\title{
INCORPORATING BIOMECHANICAL RESEARCH TOPICS INTO K-12 CLASSROOM DESIGN PROJECTS TO BROADEN PARTICIPATION AND INCREASE ENGINEERING INTEREST
}

\author{
Brandi N. Briggs, Benjamin S. Terry, Janet Yowell, and Stephanie Rivale \\ University of Colorado \\ Boulder, CO, USA
}

\begin{abstract}
This paper describes a successful new biomechanical engineering curriculum created and implemented in two highly diverse local high schools by two graduate TEAMS (Tomorrow's Engineers... creAte. iMagine. Succeed.) Fellows. In the semester-long curriculum, students create robotic surgical devices to diagnose and biopsy endometriosis, a pathology that afflicts roughly $5 \%$ of the adult female population. Curriculum focusing on anatomy, physiology, and tissue mechanics was also included to enhance the students' understanding of the human body and its response to engineering materials. Focusing this course on cutting-edge, biomechanical research that explicitly and authentically illustrates how engineering benefits society resulted in increased enrollment in engineering by underrepresented populations. This paper also discusses personal reflections by the two graduate Fellows of the benefits gained and lessons learned during the design and implementation of this innovative high school curriculum.
\end{abstract}

\section{INTRODUCTION}

In recent decades, other professions like medicine and law have made significant gains in gender representation. However, engineering continues to be dominated by Caucasian males. Women still make up less than $20 \%$ of all engineering graduates, and underrepresented minority students (URM) make up only $14 \%$ of national engineering enrollment.[1] This problem has two primary root causes. First, many students only have a vague idea of what engineers do.[2] Although we do not have national statistics, two studies reported that over $40 \%$ of the engineering students in their sample had at least one parent or relative who was an engineer.[3] This over-representation indicates that children who know someone who is an engineer are more likely to have knowledge of engineering as a career choice and go on to choose this career option for themselves. A second root cause is the lack of interest in engineering. Even though women have made significant gains in college attendance, only $1.6 \%$ of all women bachelors' degrees awarded go to women in engineering.[4] The College Board recently reported that $17 \%$ of all male SAT/PSAT takers indicated an interest in engineering, compared to only $3 \%$ of all female takers. In spite of spending roughly $\$ 400$ million dollars a year to increase public awareness of engineering, we are making few returns on this investment.[5,6] In 2008, the National Academy of Engineering (NAE) released Changing the Conversation $(\mathrm{Ct} C)$, a comprehensive study about messages and definitions of engineering most likely to increase interest in engineering in today's generation of young students.[2] The $\mathrm{CtC}$ study concluded that highlighting how engineers make a world of difference and improve the lives of people is necessary to improve both interest in and awareness of engineering. This paper describes a high-school biomechanical engineering curriculum that implements the $\mathrm{CtC}$ study suggestions through a National Science Foundation (NSF)-funded Graduate STEM Fellows in K-12 Education (GK-12) program. The GK-12 program funds graduate students to teach engineering at local area K-12 schools to prepare and develop the future engineering professoriate, to stimulate students' interest in STEM disciplines, and to help overcome the lack of diversity in engineering. 
In the University of Colorado TEAMS (Tomorrow's Engineers... creAte. iMagine. Succeed.) Program, Graduate Fellows integrate their $\mathrm{PhD}$ research into K-12 classrooms through academic year instruction, summer workshops, and the development of engineering curriculum for www.TeachEngineering.org - a free, digital library of standards-based, hands-on K-12 engineering curricula. The TEAMS Program aims to increase diversity in engineering by creating an early awareness of STEM disciplines in young people, and allows graduate students to disseminate their research to a lay audience while preparing them to enter academia with experience in teaching and developing curriculum - the overarching goal of the NSF GK-12 program. Graduate students in the TEAMS Program also gain necessary skills to enter the professoriate, including time management, teaching to diverse learning styles, public speaking, and classroom management. At the same time, young students who are typically not exposed to engineering as early learners are able to explore the possibility of engineering as a career in a fun, hands-on environment.

During the 2010-2011 academic year, two graduate TEAMS Fellows developed a new biomechanical engineering curriculum and implemented it in two highly diverse local high schools. The semester-long curriculum allows students to create robotic surgical devices to diagnose and biopsy endometriosis, a pathology that afflicts roughly $5 \%$ of the adult female population. Curriculum focusing on anatomy, physiology, and tissue mechanics was also included to enhance the students' understanding of the human body and its response to engineering materials. The course was originally taught as a trimester-long elective at a Denver-area high school, which has a $60 \%$ minority population (African American, Hispanic/Latino, and Native American). Of the students who signed up for the course, $79 \%$ were minorities, and $58 \%$ were female - statistics that are rarely seen in mechanical engineering courses. The second implementation of this course was as a semester-long STEM offering at a diverse high school in Longmont, Colorado. A shortened, 20-hour version of the course was taught during a two-week engineering summer bridge program for incoming first-year students at the University of Colorado at Boulder.

Focusing this course on cutting-edge, biomechanical research that both explicitly and authentically illustrates how engineering benefits society resulted in increased enrollment in engineering by underrepresented populations. Thus, this curriculum suggests a new approach to teaching engineering that may engage underrepresented populations and result in more meaningful outreach that could increase diversity in engineering. This paper specifically discusses the semester-long curriculum developed for Skyline High School in Longmont, $\mathrm{CO}$.

\section{CURRICULUM}

The following curriculum was developed for a semesterlong high school STEM course, with 88-minute class periods.
Ideally, prerequisites would include previous design experience, an understanding of the engineering design process, completion of algebra, and the ability to solve algebraic equations, as well as read and interpret graphs. The objective of this course is to use the engineering design process to successfully design a device to meet the requirements of a client. The course was developed to meet this objective by implementing all steps of the engineering design process. The curriculum begins with introducing the problem statement, including background information to understand the problem. A mini-lesson introduces students to engineering tools and mechanics concepts they will need to understand the environment in which their device will operate. The design process continues with brainstorming, designing, building, testing, and redesigning. Finally the students share their solution with the class during a poster presentation.

Before beginning their design project, students are introduced to the importance of keeping a lab notebook. A lab notebook helps engineers create a record of the design process so that they may preserve any data that has been collected, the testing method used to collect that data, and a dated copy of all ideas generated during brainstorming, which is essential when filing for a patent. With this in mind, engineers must write in their lab notebook for three different audiences. The first audience is the author. The engineer needs to be able to look back in her/his lab notebook to recall exactly what $\mathrm{s} / \mathrm{he}$ did and to understand any data collected. The second audience that the engineer is writing for is her/his peers. If the engineer is absent, her/his partner should be able to understand each entry in the lab notebook so that $\mathrm{s} / \mathrm{he}$ can continue to make progress on the device during the partner's absence. Finally, the engineer is writing for her/his boss or, in this case, the teacher. Lab notebooks are graded for accuracy and completeness. The teacher should be able to look at the lab notebooks at any point during the course and determine what the student completed each day. Therefore, it is required that students write in their lab notebook every day to record their progress, reflect on what they learned when they tested, and list tasks that need to be completed in the next class. This helps students to keep a steady pace throughout the semester, prompts them to regularly reflect on what they have accomplished, and keeps them focused on what needs to be completed next. Lab notebooks also prepare students for the important redesign process. Students start brainstorming on how to redesign their device to improve its performance so that they can immediately begin building during the following class periods.

\section{Problem Statement}

The problem statement is presented to the students by their client. The students are told that a local biomedical research and development firm has received a grant to develop a new surgical tool that will be used to study, diagnose, and treat endometriosis. The firm is searching for a competent engineering team to assist with the development of the tool. For this reason, the firm is contracting out the initial prototype development to various engineering teams, in this case all from 
the students' school. The teams will compete for the full contract by each developing a prototype device. The team that best meets or exceeds the objective will be awarded the contract.

The firm requires a remotely operated device that can inspect the abdominal cavity for endometriosis. Ultimately, the device will be used by surgeons who suspect endometriosis in a patient. Student teams will create and build a prototype device that will be inserted into the abdominal cavity through an incision in the umbilicus and that is remotely operated by the team members. Once inside the abdominal cavity, the device must inspect all the organs and tissue for disease. If diseased tissue is located, the device must obtain a biopsy for removal and analysis. The purpose of this device is to reduce the invasiveness of diagnosing endometriosis and provide a platform for researchers to determine the mechanism by which the disease spreads. Although beyond the scope of this course, the final version of the device will be small and robust enough to remain inside the body for at least 60 days. Each day in vivo the device will autonomously acquire images and/or video of critical anatomical regions (such as the ovaries and fallopian tubes) in order to better understand how the disease forms and spreads.

The research and development firm has specified the following requirements for a successful candidate device:

1) The device shall inflict minimal trauma to the patient during insertion. Smaller and fewer incisions heal quicker, are less prone to infection and complications, and are less painful.

2) The device shall not harm internal organs and tissue during exploration of the abdominal cavity.

3) The device must be untethered and remotely operated. Future versions of the device will remain in the body, so the prototype must not have any tethering that would prevent the entrance incision from being closed.

4) The device shall acquire digital images of the internal anatomy to confirm or disprove the existence of endometriosis.

5) If endometriosis exists, the device shall acquire a biopsy of the diseased tissue.

6) Time is of the essence during surgery. Setup, insertion, analysis and removal of the device shall not exceed 10 minutes.

For the students to successfully complete the project, they must first understand the problem statement, which means that endometriosis must be explained. Endometriosis is a disease in which the endometrium tissue, the inside lining of the uterus, grows outside the uterus in other areas of the body, causing pain, bleeding, and infertility. The tissue growth typically occurs in the abdominal cavity in the pelvic area, on the outside of the uterus, the ovaries, bowel, rectum, bladder, and peritoneal surface. It is possible, however, for the endometrium to occur in other areas of the body, such as the lungs and even arms and legs, although this is rare. The disease afflicts about five percent of women between the ages of 25 and 40 . Little is known about many aspects of the disease. For example, it is unclear how the endometrium forms in the abdominal cavity. The cause of the disease is also unknown. Further, diagnosis is difficult because endometriosis is hard to detect with ultrasound. Positive identification can occur only with laparoscopic surgery, which is invasive and expensive. During the procedure, the surgeon will visually inspect the abdominal cavity and acquire images of the afflicted region. Sometimes a biopsy will be acquired if imaging is inconclusive. Therefore, engineers are interested in developing a new method to study, diagnose, and treat endometriosis.

\section{Background}

Before brainstorming ideas, the biomechanical engineer needs to research the anatomy and physiology of the affected region and learn about the current technologies used to solve the problem. Students start the semester learning about the organization of the organs located in the abdominal cavity as well as the anatomy of the cavity, such as the different layers of tissue that make up the cavity walls. Dimensions and general shape of each organ are reviewed. Students also learn about the physiology of each organ and the abdominal cavity. A brief review of pathology and its importance to engineering is examined. Next, the students are introduced to the capabilities and limitations of laparoscopic surgery to understand where the technology is and what needs to be improved. A hands-on laparoscopic activity helps the students to understand the need for the surgical device that they are designing. Understanding the abdominal cavity and laparoscopic surgery is critical for biomechanical engineers who are designing surgical tools to replace laparoscopic surgery.

\section{Introduction to Mechanics}

The biomechanical engineer needs to understand the properties of the environment in which their device will be operating. A mini-lesson on mechanics is presented to introduce solid mechanics, fluid mechanics, viscoelasticity and tissue mechanics. During the design process, engineers must select the materials to be used in their designs. These materials must be able to withstand the forces the device will experience and must be compatible in the device's operating environment. If the device will be operating in the human body, then it must be biocompatible, survive any forces or chemicals that the body may impose on the device, and it must not harm any part of the body. The materials the engineer selects will play a very important role in the success or failure of the device. The engineer must understand the properties of the material that $\mathrm{s} /$ he selects for the device and the properties of the materials that the device will be operating on to ensure proper function.

Before learning about the mathematics that explain solid mechanics, the students experience hands-on how materials behave elastically. A typical Hooke's Law activity is explored with two different springs and several weights. Before starting the activity students are asked to describe the properties of each spring. In their notebooks, they write any differences or similarities observed, focusing on how the spring feels or behaves when stretched. The students then record the weight 
applied to each spring and the corresponding displacement. The Hooke's Law spring equation is introduced, and the students use Excel ${ }^{\circledR}$ to plot their data, calculate the spring constant of each spring, and compare the difference in response from each spring. This activity allows the students to have an in-depth understanding of how springs behave so that they can easily relate springs to elastic materials.

The solid mechanics lesson then introduces material behavior of elastic solids. Engineering stress and strain are defined and their importance in designing a device or system is explained. Instructors address how engineers measure, calculate, and interpret properties of elastic materials. Throughout the lesson, the students are encouraged to relate what they are learning to the previous activity. Often, students have difficulty understanding that elastic materials can be deformed without permanently damaging the material. Constantly relating elastic material behavior to the spring helps them to visualize this phenomenon. The students also gain a better understanding of Young's modulus, stress, and strain by directly comparing it to the spring constant, weight, and displacement seen in the activity. They can point out the similarities and differences of calculating Young's modulus versus a spring constant.

Engineers also often design devices that use fluids as lubrication, transport fluids, or have to operate in an environment that contains fluids. Therefore, it is very important for engineers to understand how fluids behave under various conditions. Understanding fluid behavior can help engineers to select the best fluid to operate in their device or to design devices that will be able to efficiently operate in an environment that contains fluids.

Students are introduced to the properties of fluids by comparing water to honey. They are asked to write in their lab notebooks any similarities and differences they notice when interacting with water and honey. Students focus their responses on the behavior of the fluid. As a class, they brainstorm what might cause these differences. The concept of viscosity is introduced. Students explore the equation for viscosity and compare this to the equation for Young's modulus. The similarities and differences in these two equations are addressed, along with the similarities and differences of the behavior of elastic solids versus viscous fluids. The methods that engineers use to determine these material properties are explained and compared to how engineers study elastic solids. Finally, several types of fluid behavior are addressed with their respective shear stress vs. rate of shearing strain diagrams. In particular, Newtonian, shear thinning, shear thickening, and Bingham plastic fluids are explained. Examples of these fluids that the students interact with in their daily lives are introduced, and how engineers use the properties of these fluids in different systems is discussed.

Biological material behavior is a combination of elastic solid properties and viscous fluid properties. This type of behavior is called viscoelasticity. Therefore, biomechanical engineers must understand viscoelastic behavior to fully characterize the behavior of biological materials. In this project, students are creating a surgical device that will operate on viscoelastic materials in the human body, so they will need to understand how the environment will react to the forces that the device imposes.

Before diving into the viscoelasticity lesson, students make three batches of silly putty, each with a different concentration of borax. They observe the properties of the material and how it reacts to forces. Each student predicts whether the silly putty behaves like a solid or fluid based on what they have learned so far. As a class, they discuss the properties of the silly putty and voice their predictions. Finally, it is revealed that the silly putty neither behaves like an elastic solid or a viscous fluid. Instead it has a combination of the properties. Therefore it exhibits viscoelastic material behavior.

The next lesson introduces the concept of viscoelasticity and describes some of the different behaviors of viscoelastic materials, including strain rate dependence, stress relaxation, creep, hysteresis, and preconditioning. Strain rate dependence is demonstrated by stretching the silly putty quickly to failure and then slowly to failure. Students note the differences in the response of the material. Viscoelastic material behavior is also compared to elastic solids and viscous fluids. Finally, the materials that have viscoelastic behavior are discussed along with the importance of engineers understanding viscoelasticity.

To demonstrate viscoelasticity, the students measure the time for the silly putty to creep six inches under its own weight. They compare the behavior of the three batches of silly putty and predict why the concentration of borax would alter the material properties of the silly putty. To further demonstrate creep, the students hang a weight on a rubber band and record the displacement of the rubber band as a function of time. They use Excel $\AA$ to plot their data and compare it to the results from the viscoelasticity lesson.

The final lesson in this curriculum describes the different components in the extracellular matrix of biological tissue and what mechanical response each component induces. In particular, the role of collagen, elastin, and proteoglycans is addressed. What each component is physically doing in each step of the stress-strain and stress relaxation diagrams is also explained.

Finally, the importance of the borax concentration in silly putty is addressed. Students learn that borax cross-links the polymer strands in glue, which forms silly putty. The amount of cross-linking is directly related to the material properties of the silly putty. More cross-linking will form a material that is stiffer and has a more solid-like behavior. Less cross-linking results in a material that is less stiff and behaves more like a fluid. This is compared to tissues in our body and the effect that collagen cross-linking has on the mechanics of the tissue.

\section{Brainstorm}

Now the biomechanical engineer has all the necessary tools to begin brainstorming and designing their device. The rules of brainstorming are established in the classroom:

1) Focus on quantity. Students should think of as many ideas as they can at this point. 
2) Withhold criticism. Never put down others or your own ideas.

3) Encourage wild ideas. This promotes creative and innovative ideas.

4) Stay focused on topic. Make sure ideas are generated to solve the problem statement.

5) Build upon the ideas of others. Engineers work as a team to form great ideas.

Start the brainstorming session with no boundaries. The students assume that they have unlimited time and budget to create the best device possible. During the following class period, the teacher introduces constraints to the problem, such as the amount of time they will have to build and the materials available to them. At this time, electronic components are distributed to each group, and the proper wiring and handling of the electronics is introduced. Each group assembles the electronics to fully understand the function and limitations of the equipment. Students continue brainstorming, focusing on realistic options for their device. Finally, the students are asked to narrow down their ideas to the top three.

\section{Design}

Students begin to develop their top three ideas by drawing a detailed sketch of each idea with labels of the materials that will be used for each part. After each of their top three ideas are thoroughly expanded, each team decides on the design they will build. The preliminary design review requires students to explain why they choose that design and why the other two designs were not sufficient.

\section{Build}

Once the teacher approves the designs, each team is given a 4-channel radio transmitter, 4-channel radio receiver, battery pack, switch, two continuous rotation servos, and two $180^{\circ}$ rotation servos. Teams also have the following materials available for the duration of the course: foam core, popsicle sticks, press board, string, masking tape, duct tape, Velcro ${ }^{\circledR}$, wooden dowels of various sizes, paper clips, pipe cleaners, Tpins, toothpicks, rubber bands, straws, index cards, and safety pins. The tools that are available for construction of the devices are scissors, Exacto ${ }^{\circledR}$ knifes, and hot glue guns.

\section{Test and Evaluate}

Testing is a crucial step of the engineering design process; however, students have a tendency to not leave enough time during the course to perform adequate testing. Checkpoints were developed in advance and strategically placed throughout the curriculum to encourage testing and to keep the students progressing at a constant rate during the course (Table 1). All testing was performed ex vivo on a bench-top simulator (Figure 1). The simulator was representative of the abdominopelvic cavity, with the small intestine made of latex tubing filled with silly putty. The simulator had a covering (black plastic) to simulate the abdominal wall. Each team had to make an incision in the abdominal wall in order to place their device inside the abdominopelvic cavity. Once inside the cavity, the device was out of view of the team members. Each device was therefore equipped with a wireless video camera to transmit the view to the team members. (Note: Wireless Color Spy Camera with PC USB Adapter by nuLime, http://www.nulime.com.)

\section{TABLE 1: SUMMARY OF CHECKPOINT OBJECTIVES}

\begin{tabular}{|l|l|l|}
\hline Checkpoint & Objective & Build Day \\
\hline One & $\begin{array}{l}\text { Critical design review: Students } \\
\text { can explain how their final design } \\
\text { will fulfill the project requirements }\end{array}$ & 5 \\
\hline Two & $\begin{array}{l}\text { Partial mobility: Devices can move } \\
\text { forward and backward in the } \\
\text { abdomen }\end{array}$ & 10 \\
\hline Three & $\begin{array}{l}\text { Full mobility: Devices can move } \\
\text { forward, backward and turn in the } \\
\text { abdomen }\end{array}$ & 15 \\
\hline Four & $\begin{array}{l}\text { "Blind" mobility: Students can } \\
\text { drive their device in the abdomen } \\
\text { with the use of the camera }\end{array}$ & 18 \\
\hline Five & $\begin{array}{l}\text { Biopsy demonstration: Devices } \\
\text { can remove one gram of diseased } \\
\text { tissue from the abdomen }\end{array}$ & 23 \\
\hline
\end{tabular}

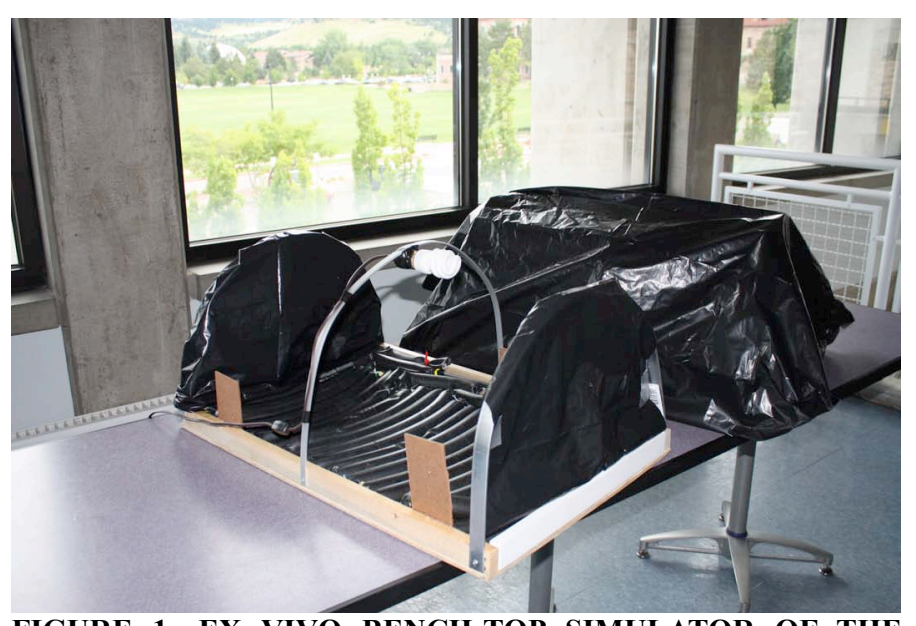

FIGURE 1: EX VIVO BENCH-TOP SIMULATOR OF THE ABDOMINAL CAVITY WITHOUT THE COVERING (LEFT) AND WITH THE COVERING (RIGHT)

Checkpoint One assesses the concept of their design. Students show progress on the chassis of their device and explain how it will move forward, backward, and turn. They describe how many of the four servos they will use and what each servo's function will be in the device. If the device is functional at this time, students demonstrate forward and backward mobility outside of the environment. This checkpoint is assessed on the fifth day of building.

On the tenth day of building, Checkpoint Two is assessed. This checkpoint requires the students to demonstrate mobility in the environment. Students must successfully navigate the entire length of the abdomen, both forward and backward. They are also required to reflect in their lab notebooks about what they learned from testing and discuss any changes that they 
need to perform to their device so that they can successfully complete this task.

Checkpoint Three adds turning within the environment to demonstrate full mobility. The device should be able to access every part of the abdominal cavity with ease at this time. Again, students reflect on the activity by explaining what they learned from testing and what they need to change to successfully complete the checkpoint or improve reliability of their device. This checkpoint is assessed on the fifteenth day of building.

On the eighteenth build day, Checkpoint Four is assessed for "blind" mobility within the environment. At this time, the skin of the patient (i.e., the black plastic covering) is put back on so that students cannot see inside of the abdomen while testing. They have to rely solely on the images captured from the video camera to navigate through the abdomen. Four mounds of different-colored Playdoh ${ }^{\circledR}$ are placed within the abdomen to represent different sites of endometriosis. The students must enter the abdomen, find all four sites of endometriosis, navigate to each site, and exit the abdomen within five minutes. They are not required to take a biopsy at this time. Students reflect in their lab notebooks on what they learned and what can be improved.

Checkpoint Five is the final checkpoint and is designed to test their biopsy device. At this point, students should be able to successfully complete all previous checkpoint tasks, which means they must also complete Checkpoint Five while solely relying on the camera for their vision. Again, four different sites of endometriosis are randomly placed in the abdomen. Students have five minutes to enter the abdomen, identify at least one of the sites of endometriosis, move their device to that site, acquire at least one gram of biopsy, and exit the abdomen. In their lab notebooks, they describe their biopsy device design, how it functions, and reflect on the checkpoint. This checkpoint is assessed on the twenty-third build day.

\section{Redesign}

After each checkpoint, students are required to reflect on what they learned from testing, focusing on how to improve their device to increase performance and reliability. This serves as the brainstorming step of the redesign process. Students then use these ideas to redesign their devices so that they can successfully complete the objectives of each checkpoint, which are required for each successive checkpoint and on the final test day.

\section{Final Test Day}

On final test day, students are required to enter the abdomen, acquire an image of all regions of endometriosis in the abdomen, collect a minimum of one gram of biopsy, and exit the abdomen in under ten minutes. Students are assessed on how many regions of endometriosis were identified; fifteen points are awarded for every region identified. The main objective of the problem statement is to identify endometriosis, so each device must be able to meet this goal to be considered successful. Five points are allocated for every $0.1 \mathrm{~g}$ of biopsy collected and twenty points for delivering the biopsy to the surgeon (the instructor). To confirm that the diseased tissue is endometriosis, it is imperative that the device be able to successfully obtain a biopsy and remove it from the abdomen. Successful removal of the device and all components is awarded fifteen points. No surgical tools or any foreign object can be left in the body after surgery.

Students have ten minutes to complete surgery. Time is of the essence; shorter surgery time means that patients need less anesthesia and less time is spent in the operating room, which is desired by both patients and surgeons. Teams are awarded one point for each minute they do not use. New surgical devices strive to achieve non-invasive procedures to reduce recovery time, risk of infection, and scarring. Therefore, the incision size is measured and one point is deducted for each centimeter. After surgery is complete, the abdomen is inspected for injury. If evidence of damage to the internal organs is present, then fifteen points are deducted. Devices used inside the body must be safe and not cause harm to any part of the body.

In their lab notebooks, students draw an outline of the abdomen and label where each region of endometriosis is identified. The ability to map diseased tissue in the abdomen will aid surgeons during the removal process of the diseased tissue. Each correct location on the map is awarded five points.

\section{Share Solution}

Students learn the importance of being able to communicate their ideas through the use of engineering drawings and a poster presentation. The basics of engineering drawings are introduced such as standard paper sizes, the difference between top, front, and side views, and properly labeling a title box. Students practice these skills by hand drawing a Lego ${ }^{\circledR}$ block with one corner cut away. Now that the students have an understanding of constructing hand-drawn engineering drawings, a 3D modeling software, Google SketchUp $\odot$, is explored. Basic tools in the program are introduced, and students create their first 3D object in the software, a chair. Students are then challenged to model their surgical device using the software. This $3 \mathrm{D}$ rendering of their device serves as an excellent communication tool to use during their poster presentations.

Poster presentations allow students to showcase their accomplishments throughout the semester. The posters are broken up into two sections: 1) a summary and description of the project and their final test day performance and 2) an advertisement of their team and product to their client. For part one, students are asked to include a description of the project and objectives, a summary of their test results on the final test day, an engineering drawing either by hand or using Google SketchUp $\mathbb{C}$ to aid in the description of their device, and a reflection of what they learned from this project. The second half of the poster is dedicated to selling their device and team to their client. Students were asked to sell their team's strengths, future improvements to their device with target test results, a sketch or 3D model of the next iteration of their device, and the amount of time and budget they will need to complete the final product. This exercise allows students to improve their 
communication skills while reflecting on the project as a whole and learning how to promote not only their accomplishments through their device but also their potential as a team.

\section{TeachEngineering}

This curriculum will be published in the National Science Foundation-funded TeachEngineering digital library (http://www.teachengineering.org) - a collection of searchable, standards-based, classroom-tested K-12 engineering curricula for use in science and math classrooms.[7] The inquiry-based lessons and hands-on activities use engineering as a vehicle for the integration of science and math, connecting real-world experiences with subjects and concepts being taught in classrooms. Mapped to educational content standards, the lessons and activities are age-appropriate, inexpensive to conduct, and relevant to children's daily lives, helping science and math come alive. The collection is a powerful resource for those in K-12 or higher education, industry and professional communities wanting to improve STEM literacy, engage young students in the joys and creativity of engineering, and increase the number of students who pursue STEM careers.

The TeachEngineering interface is notable for its standards cross-alignment in which all its lessons and activities identify specific educational standards that would be met by their use. Further, those curricular items are cross-aligned to the K-12 STEM educational standards of all 50 U.S. states, most national STEM standards and the international ITEEA standards. TeachEngineering, a National Science Digital Library (NSDL) collection, serves as a dissemination website for the curricula created from numerous National Science Foundationfunded GK-12 and RET grants to U.S. engineering colleges. Continuously growing and evolving, the collection currently contains more than 900 lessons and activities that were independently created and tested by 21 different entities, presented with a teacher-friendly, common "look and feel." Curricula created by individual authors across the nation continue to be added to the collection after meeting minimum standardized components, engineering content requirements and quality-control criteria. Each curriculum is peer reviewed by an engineer and a K-12 teacher and then edited into a standard template by a professional editor before publication.

\section{REFLECTION}

Teaching is a unique experience that many graduate students do not have the opportunity to do. It not only prepares graduate students to enter academia but also improves many of the skills needed to complete graduate research. Through the TEAMS Program, valuable lessons were learned about teaching and developing curriculum that can only be learned with experience.

Teaching as a graduate student has many benefits, especially for those aspiring to enter the professoriate. When entering academia, it is expected that one has already developed her/his teaching style and philosophy; however, very few have had the opportunity to develop their beliefs and are basing their teaching philosophy on their experiences as a student instead of as a teacher. This program builds a strong foundation for an effective teaching style and philosophy that is based on teaching experiences.

Both graduate Fellows felt that they did not have a strong teaching style or philosophy prior to entering the GK12/TEAMS Program. After completing the program, they now believe that it is imperative to teach multiple 10- to 15-minute concise mini lessons, followed by an active, hands-on learning component. This could include a worksheet, class discussion, or experiment. Switching between lessons and activities keeps the students engaged and focused during the entire class period. This teaching style also ensures that all learning styles are addressed to facilitate optimal learning. The mini lessons will engage auditory learners, while visual learners succeed through the use of illustrations and movies. Kinesthetic learners will flourish during the active, hands-on learning components. Finally, their teaching philosophy is now strongly based on including examples and projects during the course that directly relate to the students' everyday lives or benefit their local community or society as a whole. The graduate Fellows learned that the context of projects or examples used during the course strongly affects the interest and performance of students throughout the semester.

Graduate student teaching also improves many other valuable skills, such as classroom management, curriculum development, and how to ad hoc solutions to problems in the classroom. As a student teacher, it is soon learned that all the training in the world could not adequately prepare for implementing classroom management or original curriculum. It is critical to gain experience in the classroom to truly improve these skills. The graduate Fellows were able to heavily depend on their mentors to coach them through developing their own tricks in the classroom. The graduate student Fellows now have the experience that professors are expected to have, but rarely get, and can work on improving teaching skills rather than having to learn them as a new professor. Although there is much yet to learn and develop, the graduate Fellows have a great start and can continue to improve their skills.

The skills that the graduate Fellows as teachers gain from this experience also benefit their careers as graduate students. They improve essential skills in time management, problem solving, and writing and presentation skills. Having to juggle 15 hours per week of teaching, attending classes, and research results in better time management skills and increased efficiency while working. Time management is a crucial skill for graduate school and future careers in academia. Professors and graduate students have many responsibilities, so being able to work quickly and effectively will greatly enhance success in the field. Problem-solving skills improve due to the amount of quick decision making necessary in the classroom. Engineers are taught how to solve myriad challenges, so there is already a great foundation for problem solving. This opportunity enables the graduate students to put those problem-solving skills into practice, which further improves them. Finally, the graduate students became more effective communicators, which is a vital 
skill for all engineers. Teaching for a year results in a greater comfort level with public speaking. Good public speaking skills are useful when presenting research and teaching. Teachers also have to write documents for the students as well as write a detailed description of the entire curriculum so that others can teach the same course. This experience improves not only writing skills but also the ability to explain research and complicated topics to those who may not have any previous engineering knowledge. The ability to explain these topics to the general public will aid in future outreach and disseminating engineering research to the public.

Teaching and developing curriculum yields insight into lessons learned. At this age, many students have not yet learned how to learn. Knowing this, it is the teacher's responsibility to teach the students how to learn and how to recognize their own learning styles. With that in mind, the curriculum must allow students of all learning styles to thrive. The curriculum presented here has intentionally incorporated auditory, visual, and kinesthetic learning styles. In-class lectures cater to the auditory learners, pictures and demonstrations during lecture are for the visual learners, and associated activities solidify the material for the kinesthetic learners. All three aspects must be present throughout the semester so that all students have the opportunity to thoroughly learn and understand the material.

The curriculum must also be gender friendly and appeal to students of many different backgrounds and interests. Students will very quickly lose their interest, motivation and determination to succeed if the topic of the course caters only to a specific subset of students. Topics that directly relate to the students' everyday lives or that directly benefit society tend to interest a wider range of students. For students to remain motivated to successfully complete the course/project, the chosen topic must remain a constant throughout the semester.

The challenge that this poses to teachers is to continually and intentionally integrate the context of the course into everything that the students learn and every decision associated with their project. Continuing to have a context and purpose for the course and providing realistic explanations for every decision in the class increases the importance of the concepts that they learn. Every decision the teacher makes has to have a reason that relates to the problem statement. For example, the student cannot damage the testing environment because this means that they have actually just injured the "patient." This continues to engage the students and makes the project more realistic, which feeds their interest in engineering. If this is not maintained, the students will quickly forget the purpose of the curriculum and not realize the benefits of their project. This observation is consistent with both the projected outcomes of the course and the proposed recommendations from the National Academy of Engineering's Changing the Conversation study: explicitly highlighting how engineering benefits society which makes engineering more appealing to populations currently underrepresented in engineering. Interest and knowledge of engineering pre- and post-course survey data is currently being collected to quantify these results observed in the classroom.
When executing this curriculum, groups of two students work better than groups of three, four or more. Two-student teams still give them a chance to learn teamwork, yet minimizes down time and the likelihood for group members to slack. Larger group sizes tend to allow the leaders of the group to dominate the project. Less motivated and/or passive students are often pushed to the side and rarely contribute to the project, resulting in lack of interest in engineering and minimal learning. When pairing students, take into account the work ethic and personality of each student. When a strong student was paired with a weak student, it resulted in the strong student still dominating the project and taking on the majority of the workload. Weak students may be more engaged and motivated to successfully complete the project when paired with another weak student. They can no longer depend on the strong student to carry them through the project and ultimately learn more when they are with another student with similar educational motivation.

For those who are considering implementing this curriculum in their classroom, it is highly recommended that class periods be a minimum of 88 minutes. The design project associated with this curriculum requires considerable set-up and clean-up time everyday, so longer class periods allow the students to have ample build time. Finally, it is recommended that teachers have extra "intestines" on hand for when students damage them during testing. The devices should be designed to not injure the "patient"; however, throughout the semester students may accidentally puncture the intestines while exploring what their device is capable of while in the abdomen. To avoid problems during checkpoints and on the final test day, extra spans of "intestines" should always be accessible. When the abdomen is damaged, surgery time to replace the damaged portion can then be minimized.

\section{CONCLUSION}

This course attracted overrepresented numbers of both women and URM students. Of the students who signed up for the course, 79\% were minorities (where minorities make up $60 \%$ of the population) and $58 \%$ were female. We hypothesize that the increase in interest in women and minorities heavily relies on the biomedical context of the course.[8] Columbia University saw similar changes in female recruitment and retention when it changed its engineering focus on humanitarian problems. Consistent with Changing the Conversation recommendations, the context of this course explicitly focused on directly improving society and the quality of life of individuals. Often students have trouble grasping the purpose of learning math and science concepts, but this curriculum allows them to apply skills that they have learned to directly benefit someone's life. This novel curriculum also uses a cutting-edge problem that does not have a solution currently in practice. Students may feel a greater need for their project by working on a project that is current and relevant.

This curriculum, therefore, adds to the evidence base that future curriculum development and STEM education outreach 
should pick gender-friendly projects that explicitly show direct benefit to society. Professors interested in outreach are encouraged to incorporate their own research with a humanitarian focus rather than relying on the stereotypical engineering problems that are likely to attract the same traditional population we are trying to diversify.

\section{ACKNOWLEDGMENTS}

This material is based upon work supported by the National Science Foundation under grant no. DGE 0946502. The authors would like to acknowledge the support and feedback of the Skyline High School STEM Academy teachers and leadership, especially those of Scott Duckworth who assisted the Fellows in course implementation. The authors would also like to thank Denise Carlson for her assistance with this paper.

\section{REFERENCES}

[1] NSF, "Women, Minorities, and Persons With Disabilities in Science and Engineering," National Science Foundation, Arlington, VA, 2009.

[2] NAE, Changing the Conversation: Messages for Improving Public Understanding of Engineering. Washington, D.C.: National Academies Press, 2008.

[3] R. M. Wentling and C. Camacho, "Women Engineers: Factors and Obstacles Related to the Pursuit of a Degree in Engineering," Journal of Women and Minorities in Science and Engineering, vol. 14, p. 50, 2008.

[4] Engineering Workforce Commission, "Engineering and Technology Degrees," Washington, DC, 2006.

[5] NAE, Ed., Raising public awareness of engineering. National Academy Press, 2002.

[6] K. Edwards and E. A. Sawtell, "College-Bound Students' Interest in Engineering: Pathways and Characteristics of the Precollege Pipeline " presented at the American Educational Research Conference, New Orleans, LA, 2011.

[7] J. F. Sullivan, M. N. Cyr, M. A. Mooney, R. F. Reitsma, N. C. Sha, M. S. Zarske, and P. A. Klenk, "The TeachEngineering Digital Library: Engineering Comes Alive for K-12 Youth," Proceedings, ASEE Annual Conference, Portland, OR, June 2005.

[8] B. S. Terry, B. N. Briggs, and S. Rivale, "Work in Progress: Gender Impacts of Relevant Robotics Curricula on High School Students' Engineering Attitudes and Interest," Proceedings, ASEE/IEEE Frontiers in Education Conference, Rapid City, SD, 2011. 